\title{
Mobile Motion: Multimodal Device Augmentation for Musical Applications
}

\author{
Matt Benatan, lan Symonds and Kia Ng \\ School of Computing, School of Electronic and Electrical Engineering and School of Music \\ ICSRiM, University of Leeds, United Kingdom \\ mobilemotion@icsrim.org.uk
}

\begin{abstract}
Mobile devices have become an integral part of $21^{\text {st }}$ century lifestyle. From social networking and business to day-to-day scheduling and multimedia applications, smartphones and other portable handsets are now the go-to devices for interaction in the digital world. Currently, mobile devices typically utilise direct user interfaces such as touch screens, where interactions are performed directly by controlling graphical elements or controls on the interface. This project looks to bring device interaction out of the virtual world and into the physical world. With a 'free-gesture' approach, portable applications can break away from the virtual world, enabling the mobile platform to be harnessed as a physical augmented interface for musical performance, education, medical research and beyond.
\end{abstract}

Mobile. Motion. Multimedia. Electronics. Interactive. Multimodal. Sensor. Music.

\section{INTRODUCTION}

Over recent years mobile devices have become central to the way many users experience the web, multimedia and beyond. These devices rely largely on touch-based interfaces; often with other sensors, such as accelerometers, being used for more novel forms of user input. Through augmenting existing technologies with positionaware sensor capabilities, this project looks to further explore mobile device interaction, and enable users to engage with virtual technologies through physical gestures. This has already proven to be hugely successful in the realm of gaming, with products such as Microsoft's Kinect (Shotton et al., 2011) and the Nintendo Wii (Nintendo, 2011).

The current limitation for motion control on the mobile platform is largely due to the fact that existing technologies rely on static hardware units to provide a point of reference - something that poses a challenge for mobile devices since they cannot rely on stationary components. Through this project we are developing a prototype to address this limitation.

To ensure that the project results in a system that positively enhances the user interaction experience, key requirements include:
- Precision: the system should be able to consistently provide data on the location of the device relative to the user with sufficient accuracy;

- Portability: the physical gesture mechanism should be capable of working anywhere the device goes, without being bound to stationary hardware;

- Low latency: the system should respond to user interaction within an acceptable timeframe (depending on the application context), ensuring a smooth and natural response.

\section{EXISTING DEVELOPMENTS}

Although there is a vastly expanding range of smart-phones and mobile devices, none of them offer a comprehensive local positional tracking mechanism; however they are all equipped with a global tracking mechanism, GPS. In order to survey current trends in local tracking systems, a number of motion control technologies are discussed in this section.

One similar commercially available product is the Nintendo Wii (Nintendo, 2007 \& 2011), as it combines physical input with accelerometer and infrared positioning sensors to provide a gesturebased control system. The principal behind the 
motion detection component is simple: a sensor bar is placed in a fixed position, which then emits two infrared beams. This is then picked up by the Wii remotes' camera, and the information processed to triangulate the position of the remote in relation to the sensor bar, thus providing motion tracking.

Following in Nintendo's footsteps, the two other major console developers, Sony and Microsoft, have now also recently released motion control interfaces. Each of these uses a slightly alternative approach. In Sony's case, their PlayStation Move system (Mikhailov, 2009) relies on tracking an LED orb. To enable it to work in a range of environments, the colour of the orb changes dynamically to allow it to be easily distinguishable by the camera. The controller also houses an accelerometer, a rate sensor and a magnetometer, which provide further gestural information. To enable the controller to function in three dimensions, Sony's PlayStation Eye (the camera) determines the distance of the controller from the camera via the size of the image corresponding to the orb.

Microsoft has further developed the visual light approach through their Kinect system (Shotton et al., 2011). Unlike the Wii and PlayStation Move, this system does not require any form of controller, and instead relies on depth imaging. This system is able to follow user movement through the implementation of body model and gesture analysis algorithms. Thus, the system is capable of recognising and tracking parts of the users, such as hands and heads.

Although there are currently no portable local motion tracking systems for the mobile environment, there have been a number of developments in the area of multimodal mobile device interaction. One system, such as the Multimodal Home Entertainment Interface developed by Gruenstein et al (2008), uses mobile device interaction within a home entertainment environment. This system utilises speech input from the user, as well as touch-based interaction, to navigate a television programme guide.

With the current advancements in sensor technology, there has been increased interest in sensor-based gesture interfaces in a wide range of research applications, such as Young's work on the Hyperbow (Young, 2003). Through the use of accelerometers and strain sensors, Young developed a system capable of measuring the bowing gestures of violinists. This enabled gesture pattern data to be used for a range of purposes, including pedagogical applications.

These examples demonstrate the interests and trends in creative new developments in the field of sensor-based user interaction. Gesture-based motion control is a natural and engaging approach (Wilson, 2000), with great potential to be adapted to a vast variety of applications; with a diverse range of industries, including education and entertainment.

\section{DESIGN AND DEVELOPMENT}

\subsection{Sensor survey}

In order to develop a prototype system, a suitable method of sensor augmentation had to be chosen. To do so, a number of motion-control systems were surveyed.

\subsubsection{Infrared}

A popular example of infrared motion-detection technology is the Nintendo Wii (Nintendo, 2007). The Wii, though successful within home gaming applications, has certain limitations for portable devices. The primary weakness here is its susceptibility to interference from other light sources, such as candle or sun light, in certain environments. As such, infrared systems require specific lighting environments to perform consistently, and would thus work inefficiently outdoors, making it a poor choice for a mobile device system.

\subsubsection{Near Magnetic Field Coupling}

Another approach considered was to use near magnetic field coupling, as demonstrated by $M$. Bezdicek's hand tracker (Bezdicek \& Caldwell, 2006)]. This system provides highly accurate coordinates of finger positions and transmits them via Bluetooth, thus making it an appropriate approach for hand-specific gestures within computer interaction. However, with the effective range of near magnetic field coupling being approximately $15 \mathrm{~cm}$, this approach would not satisfy the distance requirements in the case of this project.

\subsubsection{Ultrasonic Sensors}

A popular technique for acquiring positional information within robotics is through the use of ultrasonic transceivers. This approach is used for a broad variety of range-finding applications, and has a number of qualities that make it ideal for use in this project. The performance of ultrasonic devices varies according to the frequency used. There are a range of ultrasonic sensors available; the most common being $40 \mathrm{kHz}$ and $125 \mathrm{kHz}$ transducers. Due to the short wavelength emitted by the $125 \mathrm{kHz}$ models, the signal dissipates rapidly, resulting in a range of approximately $10 \mathrm{~cm}$. As such, the $40 \mathrm{kHz}$ model was used, as it provides an effective range of up to $150 \mathrm{~cm}$; thus fulfilling the range requirements of this project. 
Ultrasonic sensors are also far less susceptible to noise than infrared, as the ultrasonic receiver is tuned to receive within a narrow frequency band, resulting in a particularly high level of noise rejection. These factors make ultrasound an attractive option when compared to other available technologies, providing both the necessary range and accuracy required. Additionally, the ultrasonic components are small, light weight and can be easily powered by batteries, making them highly portable.
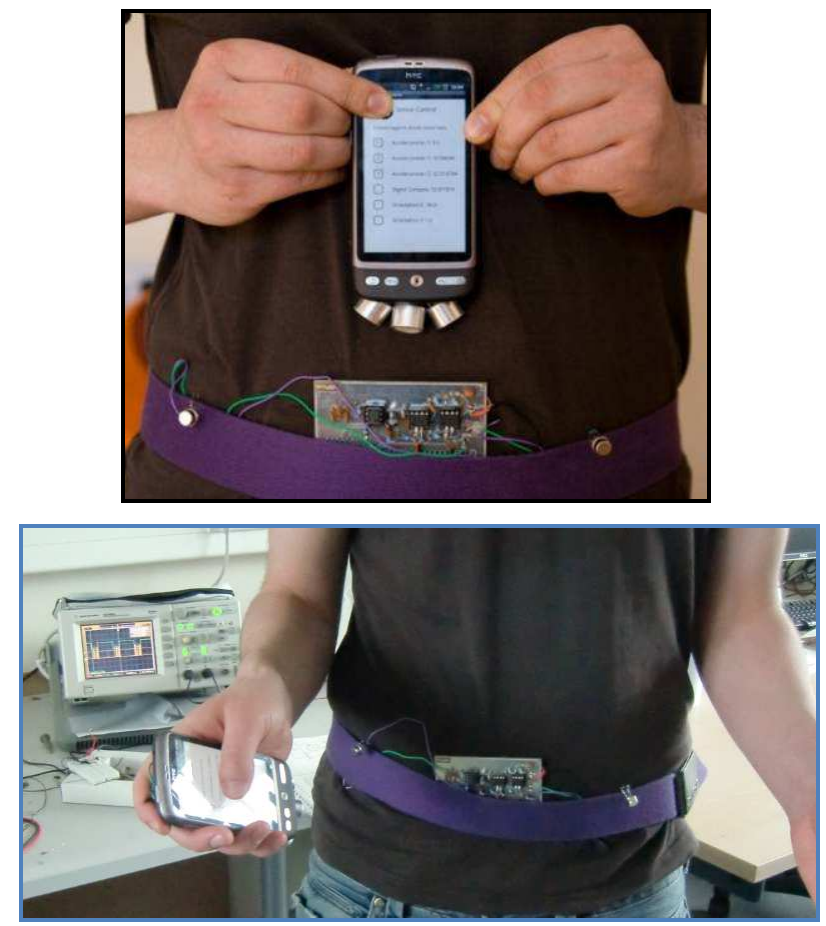

Figure 1: Mobile Motion prototype.

In order to provide user-relative positional tracking, the system required one ultrasonic transmitter, two ultrasonic receivers and a radio system. The receivers are worn on the body of the user, and the transmitter attached to the device. This enabled the ultrasonic signal's time of flight to be calculated relative to the two receiver points.

\subsection{Ultrasonic distant measurement}

The system works by sending $50 \mathrm{~Hz}$ bursts of $40 \mathrm{kHz}$ ultrasonic signals from a transmitter array (see Figure 1). $50 \mathrm{~Hz}$ was chosen as the optimal burst frequency as it was the highest frequency tested to maintain a low error rate. The same $50 \mathrm{~Hz}$ timer also triggers the transmission of a radio signal. The bursts are then intercepted by the two receiver units, and their time of arrival is compared with the radio signal on the Arduino board (http://www.arduino.cc).

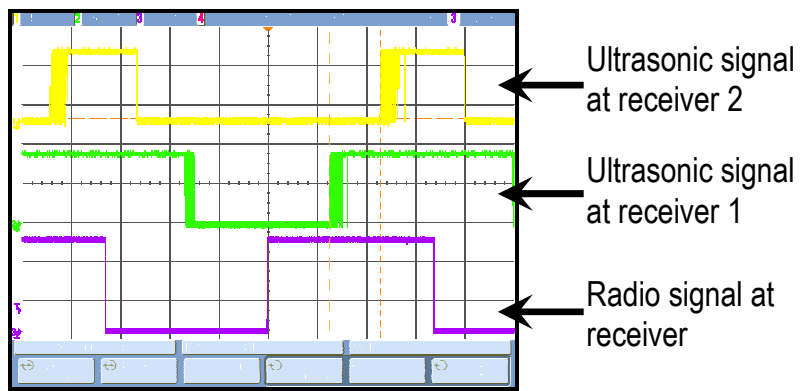

Figure 2: Signal trace using an oscilloscope

Figure 2 illustrates the different positions of the units resulting in the ultrasonic pulse reaching the receivers at different times. The position of the transmitter is then calculated through comparing their time of interception with the radio signal. From this, the propagation time for each ultrasonic signal can be determined, and the position of the transmitter calculated.

Figure 3: Ultrasonic sensor setup.

\subsection{Accessing mobile device sensors}

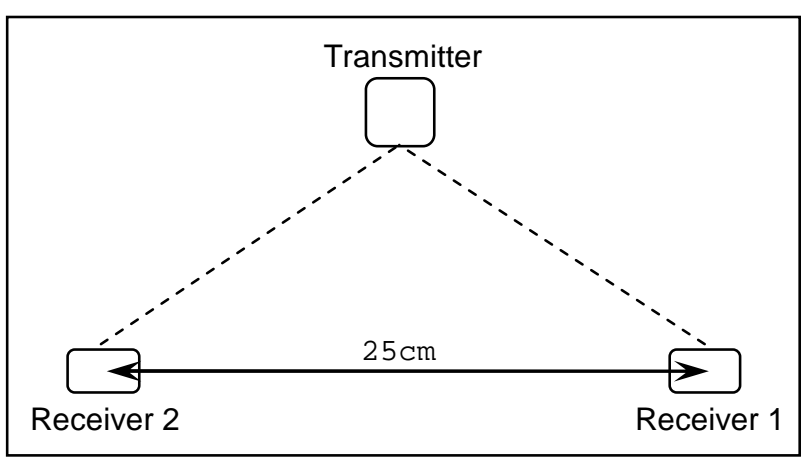

The mobile device's onboard accelerometer is key to providing information on the forces acting on the device, and thus essential for providing another representation of gesture information. In order to access this information, a custom Android application was developed.

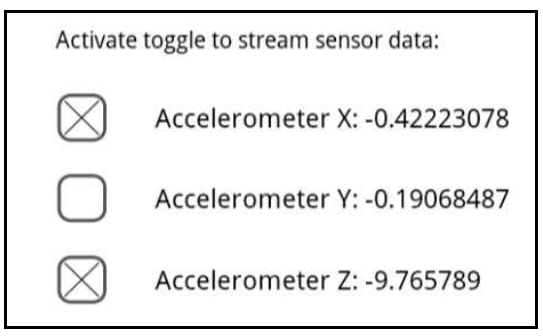

Figure 4: Sensor Control Android app. screenshot.

The application, Sensor Control (freely available via mobilemotion.leeds.ac.uk), streams the device's sensor measurements wirelessly via UDP. These are then intercepted and processed by a computer for real-time processing, used to collate and interpret the data within the prototype system. 


\subsubsection{Gesture recognition}

Once the sensor information could be streamed from the mobile device, it is possible to analyse the data for the development of gesture following algorithms. These algorithms could then be used to identify specific gesture patterns and trend and use them to trigger actions within the software for multimedia mapping. Gestural following techniques are also central to reducing latency; through detecting the gesture onset, the system is able to dynamically predict the motion, rather than waiting for the entire gesture to be enacted.

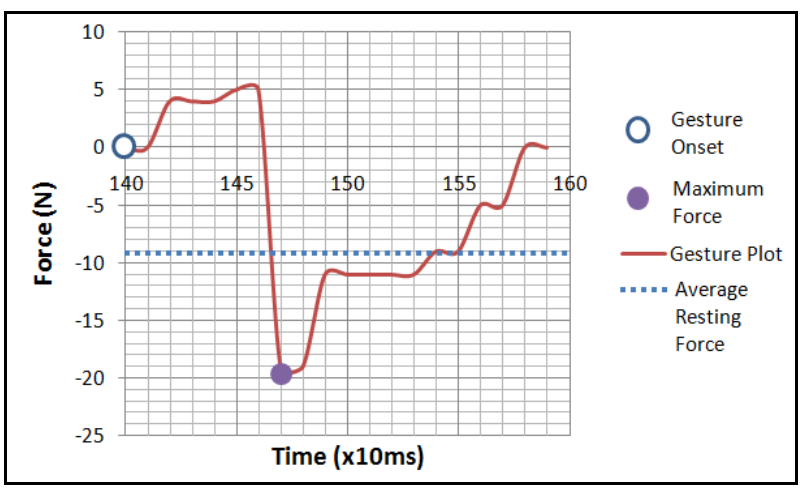

Figure 5: Graph depicting forces acting on the accelerometer sensor's $x$-axis.

\subsection{Latency}

One of the key factors in developing a successful real-time interface is response time. As discussed by Meier in the Google I/O 2010 conference (Meir, 2010), mobile device interaction is extremely time critical, and users have come to expect efficient, low-latency performance from their devices. To ensure latency is kept within acceptable boundaries, the performance of existing motion control applications has been used as a guideline. Tests on the most advanced commercial systems, Move and Kinect, demonstrated that these systems have a typical average latency of $100 \mathrm{~ms}$, up to $200 \mathrm{~ms}$ in some cases (Leadbetter, 2010).

\subsubsection{Latency testing}

In order to provide an accurate figure for the system latency, each subsystem was tested individually, from which the overall latency could be determined.

Currently, results demonstrate that the ultrasonic positioning system exhibits a maximum latency of approximately $32 \mathrm{~ms}$ (including software and hardware processing), with the accelerometer system performing at around $40 \mathrm{~ms}$ (including data transfer and software processing). As the ultrasonic positioning data is only accessed when triggered by the phone sensors, the accelerometer is the primary contributor to system latency. As such, the average system latency has been observed to be $48 \mathrm{~ms}$.

\subsection{Prototype testing}

To provide a platform for qualitative testing, a virtual xylophone program was conceived to enable the user to trigger xylophone audio samples using the mobile device as a virtual mallet. The software uses ultrasonic data to compute the position of the device in relation to the user, increasing the note pitch to the right, and decreasing it to the left, just as with a real xylophone. The trigger (hit) and velocity (force) data are determined using the device's accelerometer; allowing the user to virtually tap gently for softer sounds, and harder for louder feedback. The audio samples are then triggered accordingly, providing a realistic aural reaction to the user's virtual interaction.

\section{CONCLUSION AND FURTHER WORK}

The paper presents current work on the design and development of a position-aware motion control interface. The multi-modal approach has proven that ultrasonic sensors can be used to augment existing devices to enhance their capabilities and produce a usable interactive system. The system is currently being evaluated with an application scenario for a virtual xylophone, simulating and controlling audio samples recorded during the Ruskin Rocks project (Ng, 2008).

Future work will focus on improving the efficiency of the gesture following and recognition system, and on further optimisation.

This technology also lends itself to a range of other applications. As a gesture analysis tool, it could prove useful for studying the movement patterns of percussionists and conductors; an application that could be used in a similar way to the i-Maestro project for music pedagogy (Ng, 2008). Furthermore, networking the device could effectively create a virtual stage - whereby a number of people could interact with a variety of virtual instruments and/or share one virtual instrument. With further development, it would also be possible to combine this system with other sensing and tracking technologies, including global positioning systems, to create a connected world of augmented gesture communication.

\section{REFERENCES}

Bezdicek, M., Caldwell, D. (2006) Portable Absolute Position Tracking System for Human Hand Fingertips. Proceedings of Virtual Concept 2006, Cancún, Mexico, 27 November - 1 December 2006. 
Gruenstein, A., Hsu, B., Glass, J., Seneff, S., Hetherington, L., Cyphers, S., Badr, I., Wang, C., Liu, S. (2008) A Multimodal Home Entertainment Interface via a Mobile Device. Proceedings of the 9th SIGdial Workshop on Discourse and Dialogue, Columbus, Ohio, USA, 19-20 June 2008, pp.1129. Association for Computational Linguistics, Stroudsburg, PA, USA.

Leadbetter, R. (2010) Tech Analysis: Kinect. http://www.eurogamer.net/articles/digitalfoundrykinect-tech-analysis (31/03/2011).

Meir, R. (2010) A Beginner's Guide to Android. Google I/O 2010, San Francisco, USA, 19-20 May 2010. Session Video.

http://www.google.com/events/io/2010/sessions.html (31/03/2011).

Mikhailov, A. (2009), PlayStation Motion Controller Interview Part 2.

http://www.viddler.com/explore/sceablog/videos/546/ (27/05/2011).

Ng, K. (2008) Technology-Enhanced Learning for iMaestro Framework and Tools. Electronic
Visualisation and the Arts, London, UK, 22-24 July 2008. British Computer Society, London.

Nintendo (2007) What is Wii?

http://web.archive.org/web/20080212080618/http:// wii.nintendo.com/controller.jsp (31/03/2011).

Nintendo of Europe GmbH (2011) Wii - Nintendo. http://wii.nintendo.co.uk (31/03/2011).

Shotton, J., Fitzgibbon, A., Cook, M., Sharp, T., Finocchio, M., Moore, R., Kipman, A., Blake, A. (2011) Real-Time Human Pose Recognition in Parts from Single Depth Images. IEEE Computer Vision and Pattern Recognition 2011, Colorado, USA, 21-25 June 2011. IEEE, Colorado, USA.

Wilson, F. (2000) The Hand. Vintage Books, New York, NY, USA.

Young, D. (2003) Wireless Sensor System for Measurement of Violin Bowing Parameters. Proceedings of Stockholm Music Acoustics Conference, Stockholm, Sweden, 6-9 August 2003. KTH Speech, Music and Hearing, Stockholm, Sweden. 